\title{
Cardiovascular risk factor in MPN patients
}

\author{
Vincenzo Accurso $^{1}$ D $\cdot$ Marco Santoro $^{2} \cdot$ Salvatirce Mancuso ${ }^{1} \cdot$ Sergio Siragusa ${ }^{1}$
}

(c) Springer Science+Business Media, LLC, part of Springer Nature 2020

To the Editor,

Fernanda Salles Seguro et al. recently published an article entitled "Risk factors and incidence of thrombosis in a Brazilian cohort of patients with Philadelphia-negative myeloproliferative neoplasms" [1], describing, with a retrospective analysis, the thrombotic and hemorrhagic events that occurred in a cohort of 334 Brazilian patients with $\mathrm{Ph}-$ negative chronic myeloproliferative diseases (MPNs) [2].

In this report the authors claim that the revised International Prognostic Score in ET for thrombosis (r-IPSETt) "stratifies patients in up to four risk categories using four variables: $\mathrm{CV}$ risk factors, age $>60$ years, thrombosis history and JAK2 V617F". Actually, this classification does not take into account the cardiovascular risk factors (CVR) which were instead assessed in the previous IPSET-t score [3]. CVR aren't taken into account for polycythemia vera (PV) and myelofibrosis (MF). For patients with PV the thrombotic risk is currently assessed with the old traditional classification that distinguishes low-risk and high-risk patients, according to whether they are more or less than 60 years-old and to previous history of thrombosis.

To date, there is no validated score for thrombotic risk in MF. However, there is evidence that one or more CVR such as cigarette smoking, hypertension, obesity, diabetes and dyslipidemia can increase thrombotic risk in MPN [4].

In a previously proposed thrombotic risk classification model, to the traditional high and low-risk category was added an intermediate-risk category, collecting all the patients aged under 60 , with no history of thrombosis, but with the presence of CVR. This thrombotic risk classification model is almost not used in clinical practice [5].

Vincenzo Accurso

casteldaccia@tiscali.it

1 Hematology Divisione, University Hospital Policlinico "Paolo Giaccone", Palermo, Italy

2 Dept of Surgical, Oncology and Stomatological Disciplines, University of Palermo, Palermo, Italy
Cerquozzi et al. explored the association of CVR with the occurrence of arterial or venous events at or following diagnosis; they found that older age ( $\geq 60$ years), hypertension, diabetes, hyperlipidemia, and normal karyotype were associated with arterial events, whereas younger age ( $<60$ years), female sex, palpable splenomegaly, and history of major hemorrhage were associated with venous events [6]. In a recent report by Barbui et al. it is highlighted as hypertension in low-risk patients with PV increases the risk of arterial thrombosis [7].

Today for patients with PV or essential thrombocythemia (ET) with less than 60 years and with one or more CVR, there is no indication for cytoreductive therapy. In 2018 Barbui et al. in "Philadelphia chromo-some-negative classical myeloproliferative neoplasms: revised management recommendations from European LeukemiaNet" [8], recommended that "general risk factors for thrombosis, including smoking habit, diabetes mellitus, arterial hypertension, and hypercholesterolemia, should also be considered". On the basis of these recommendations, we studied the frequency of CVR in a cohort of 603 patients with MPN followed from January 1997 to December 2019 at the center for chronic myeloproliferative diseases of our hematology division. We calculated the frequency of cigarette smoking, arterial hypertension, diabetes, dyslipidemia and obesity in 138 cases of PMF (Overt myelofibrosis or Myelofibrosis post ET an post PV) 48 of prefibrotic myelofibrosis (prePMF), 169 of $\mathrm{PV}$ and 249 of ET. We also distinguished patients with a single cardiovascular risk factor from those with multiple risk factors. The results are described in Table 1. Overall CVR are present in 458 patients with MPN (75.95\%). 245 patients (40.63\%) have only one cardiovascular risk factor, while 213 (35.32\%) have more than one cardiovascular risk factor. Patients with CVR have a median age of 67.57 years, while those without risk factors show a median age of 53.98 years $(\mathrm{p}<0.001)$.

The high frequency of CVR in patients with MPN rises significant questions regarding the prognosis and therapeutic choices. In our opinion, however, there are no conclusive data in this regard and prospective studies are needed to 
Table 1 Cardiovascular risk in 603 MPN patients

\begin{tabular}{llcrr}
\hline CVR & PMF no 138 & PrePMF no 48 & PV no 169 & \multicolumn{1}{c}{ ET no 249 } \\
\hline Smoke & $20(14.49 \%)$ & $11(22.91 \%)$ & $25(14.79 \%)$ & $35(14.05 \%)$ \\
Hypertension & $87(63.04 \%)$ & $26(54.16 \%)$ & $107(63.31 \%)$ & $159(63.85 \%)$ \\
Diabetes & $23(16.66 \%)$ & $5(10.41 \%)$ & $29(17.15 \%)$ & $35(14.05 \%)$ \\
Dyslipidemia & $13(9.42 \%)$ & $7(14.58 \%)$ & $47(27.81 \%)$ & $63(25.30 \%)$ \\
Obesity & $11(7.97 \%)$ & $4(8.33 \%)$ & $13(7.69 \%)$ & $22(8.83 \%)$ \\
\hline
\end{tabular}

determine the thrombotic risk in patients with chronic myeloproliferative $\mathrm{Ph}$-negative disease with and without CVR.

If it was clearly demonstrated that CVR lead to a significant increase in thrombotic risk, the use of cytoreductive therapy may also be considered in these patients.

\section{Compliance with ethical standards}

Conflict of interest The authors declare no conflict of interest.

\section{References}

1. Seguro FS, Teixeira LLC, da Rosa LI, da Silva WF (2020) Risk factors and incidence of thrombosis in a Brazilian cohort of patients with Philadelphia-negative myeloproliferative neoplasms. J Thromb Thrombolysis. https://doi.org/10.1007/s11239-01902029-y

2. Arber DA, Orazi A, Hasserjian R et al (2016) The 2016 revision to the World Health Organization classification of myeloid neoplasms and acute leukemia. Blood 127:2391-2405
3. Santoro M, Accurso V, Mancuso S, Carlisi M et al (2019) Comparison between thrombotic risk scores in essential thrombocithemia in essential thrombocythemia and survival implications. Hematol Oncol 37(4):434437

4. Accurso V, Santoro M, Mancuso S, et al (2020) Cardiovascular risk in essential thrombocythemia and polycythemia vera: thrombotic risk and survival. MJHID 12(1):e2020008

5. Finazzi G, Barbui T (2005) Risk-adapted therapy in essential thrombocythemia and polycythemia vera. Blood Rev 19:243-252

6. Cerquozzi S, Barraco D et al (2017) Risk factors for arterial versus venous thrombosis in polycythemia vera: a single center experience in 587 patients. Blood Cancer J 7:662

7. Barbui T et al (2017) ASH 2017. Blood

8. Barbui T, Tefferi A, Vannucchi AM et al (2018) Philadelphia chromosome-negative classical myeloproliferative neoplasms: revised management recommendations from European Leukemia. Net Leukemia. 32(5):1057-1069

Publisher's Note Springer Nature remains neutral with regard to jurisdictional claims in published maps and institutional affiliations. 